\title{
80Gb/s Multi-wavelength Optical Packet Switching using PLZT Switch
}

\author{
Katsuya Watabe ${ }^{1}$, Tetsuya Saito ${ }^{2}$, Nobutaka Matsumoto ${ }^{1}$, \\ Takuo Tanemura ${ }^{3}$, Hideaki Imaizumi ${ }^{3}$, Abdullah Al Amin ${ }^{4}$, \\ Mitsuru Takenaka ${ }^{4}$, Yoshiaki Nakano ${ }^{4}$, and Hiroyuki Morikawa ${ }^{3}$ \\ ${ }^{1}$ Graduate School of Frontier Sciences, The University of Tokyo \\ Kashiwanoha 5-1-5, Kashiwa-shi, Chiba, 277-8561 JAPAN \\ ${ }^{2}$ Graduate School of Science and Engineering, Chuo University \\ Kasuga 1-13-27, Bunkyo-ku, Tokyo, 112-8551, JAPAN \\ ${ }^{3}$ School of Engineering, The University of Tokyo \\ Hongo 7-3-1, Bunkyo-ku, Tokyo, 113-8656, JAPAN \\ ${ }^{4}$ Research Center for Advanced Science and Technology, The University of Tokyo \\ Komaba 4-6-1, Meguro-ku, Tokyo, 153-8904, JAPAN \\ k_watabe@mlab.k.u-tokyo.ac.jp \\ http://www.hotaru-project.net/
}

\begin{abstract}
This paper proposes $80 \mathrm{~Gb} / \mathrm{s}$ multi-wavelength optical packet switching(OPS) using a PLZT switch. The Multi-wavelength OPS Network can achieve low implementation costs compared to existing OPS networks in which the number of wavelengths is large. In this network, the header is processed separately from the payload. The payload is divided into multiple segments. Each segment is then encoded into different wavelengths. After assignment of wavelength to each payload segment, payload segments are multiplexed into an optical signal which is then transmitted through an optical fiber. Therefore, the number of components required for intermediate node functionality stays the same for the number of wavelengths used for payload transmission. This paper shows a fundamental experiment of the Multi-wavelength OPS network using a PLZT switch. PLZT switch provide a fast optical switching with low noise, independent polarization, and low drive voltage. In this paper, we describe the detail of the experiments and results.
\end{abstract}

Keywords: multi-wavelength, WDM, optical packet, PLZT switch

\section{Introduction}

Wide deployment of broadband Internet access and new multimedia services such as real-time streaming have caused enormous traffic growth in the Internet. It is expected that this traffic will keep increasing with the emergence of new online services such as on-demand service for high-definition movies.

For transmission of large traffic volumes, the WDM technology has been widely researched and a number of test-bed systems have been developed recently[1]. According to studies in [2], the capacity of transmission within a single fiber can exceed $10 \mathrm{~Tb} / \mathrm{s}$. 
In addition, the optical packet switching (OPS) network technology has been researched in order to achieve high bandwidth utilization and low power consumption as well as to minimize the number of devices in the WDM network $[3,4]$. However, the existing OPS network technologies transmit the whole packet that consists of the header and the payload through a single wavelength. This functionality requires intermediate nodes to have necessary components to support it. The node must have label processing units and contention resolution units for each wavelength. This will cause the number of devices for construction of WDM networks to grow with each additional wavelength [5].

In order to solve the problem, the Multi-wavelength OPS Network Technology has been researched for OPS networks to achieve scalability with the number of wavelengths. In contrast with the existing OPS technologies, the header of the packet is processed separately from the payload. The header is encoded and transmitter at dedicated wavelengths. The payload itself is divided into multiple segments which are encoded and transmitted at different wavelengths. Therefore, this requires core nodes to contain necessary components for each port, not for each wavelength.

The feasibility of the multi-wavelength OPS technology has been confirmed with Semiconductor Optical Amplifier (SOA) switch and $\mathrm{LiNbO}_{3}(\mathrm{LN})$ switch $[6,8]$. However, it has never been confirmed with a PLZT switch which has the capabilities of fast switching, low noise, polarization independency, and low drive voltage.

In this paper, we experiment the multi-wavelength OPS technology using a PLZT switch in order to evaluate its feasibility. In this expetiment, we demonstrate $80 \mathrm{~Gb} / \mathrm{s}(10 \mathrm{~Gb} / \mathrm{s} \times 8$ wavelengths $)$ multi-wavelength OPS using a PLZT switch and measure Bit Error Rate (BER) and the eye pattern.

This paper is organized as following. Section 2 describes details of multiwavelength OPS technology. Section 3 explains the experimental setup and its detail, and Section 4 shows the experimental result. Finally, we conclude this paper in Section 5 .

\section{Multi-wavelength OPS Network}

This section describes the characteristics of the multi-wavelength OPS network with comparison to the existing OPS networks.

In the existing OPS network, every packet consisting of the header and the payload is encoded into individual wavelength. Therefore, after input optical signal is demuxed into multiple wavelengths, independent processing modules are required for each wavelength in order to perform necessary functions for packet switching such as packet switching, wavelength conversion, and contention resolution as illustrated in Fig.1. Therefore, this property will not provide good scalability for the larger number of devices because of the cost, the physical size of the system and the complexity for controlling the node in the future WDM network with the large number of wavelengths. 


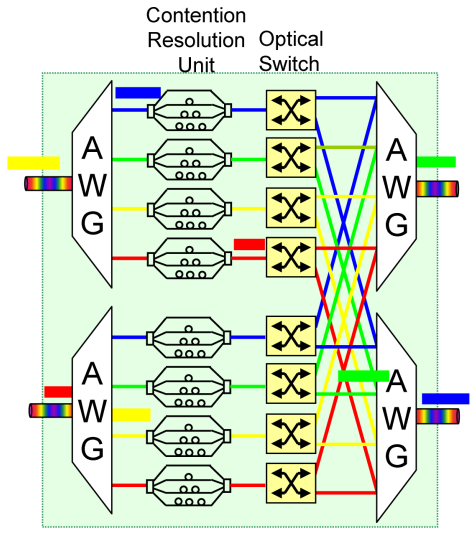

Fig. 1. Node Architecture Diagram of Traditional OPS Networks

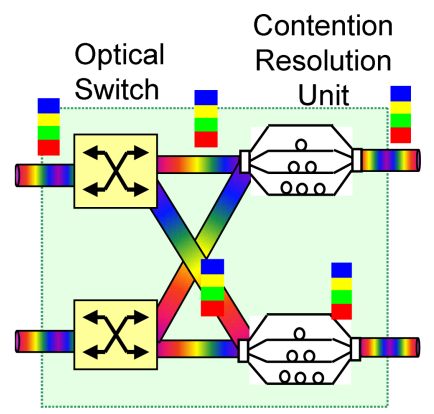

Fig. 2. Node Architecture Diagram of Multi-wavelength OPS Networks

In contrast to that, in the multi-wavelength OPS network, each packet is encoded into multiple wavelengths as illustrated in Fig.2. The header is encoded into one wavelength. The payload is divided into multiple segments. Each segment is encoded into different wavelengths. At the intermediate node in this network, the payload is forwarded in accordance with the header information. Therefore, when the number of wavelengths for the payload increases, the number of devices in the intermediate node does not change.

In the multi-wavelength OPS network, the payload can contain any data in the traditional OPS network. However, due to the characteristics that the payload in the multi-wavelength OPS network is encoded into multiple wavelengths, the composition of the payload influences the implementation cost and the characteristics of the network.

Currently two methods of the composition have been proposed: 1) the payload contains multiple packets of upper layer protocols[6], and 2) the payload contains one packet of upper layer protocols[7] as illustrated in Fig.3 and Fig.4 respectively. 


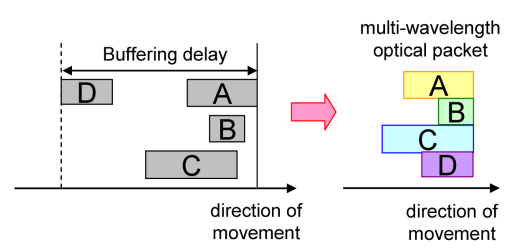

(a) Characteristic of Buffering delay

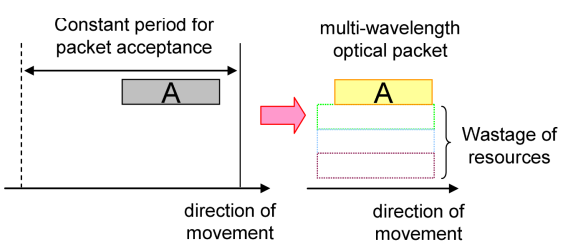

(b) Characteristic of wavelength wastage

Fig. 3. Payload containing multiple packets of upper layer protocols

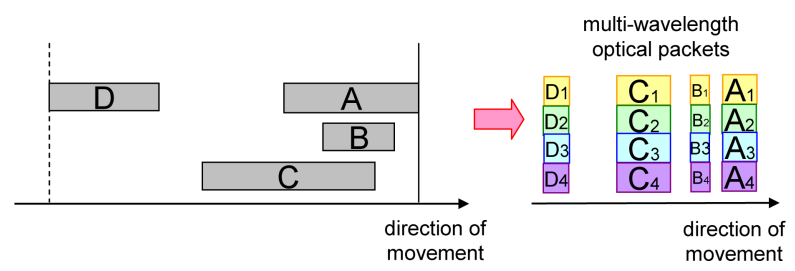

Fig. 4. Payload containing a single packet of upper layer protocols

With the former method, incoming packets of upper layer protocols are buffered at an ingress edge node for defined time period. The buffered packets which have the same destination form a payload. The maximum number of the packets in the buffer is equal to the number of available wavelengths. Hence, when the number of the packets with the same destination in the buffer reaches the number of the wavelengths, the payload is sent so that each upper layer packet from the payload is transmitted on separate wavelength. This method does change the structure of the upper layer packet so fragmentation and reassembly of those packets are unnecessary. Therefore, it makes the composition of the edge node simple. However, the time period for buffering affects the jitter. In addition to that, the utilization of wavelengths is low when the number of packets towards the same edge node does not reach the number of the wavelengths within defined time period. The feasibility of the multi-wavelength OPS network with this method has been confirmed by Onaka et al.[6]. Their node architecture was based on broadcast-and-select switch which consists of SOA switches with multistage configuration.

In contrast with described method, the second method uses only one single upper layer packet for the payload. Namely, a single upper layer packet is divided into multiple segments. The advantage of this method is that the ingress edge node transmits immediately upon packet reception. In the previous method, a node must wait until the buffer will receive enough packets for the payload or go by defined time period. In addition, the utilization of wavelengths is higher than one of the former method. This is because in the method with packet fragmentation, the available wavelengths for the payload are always utilized. However, the implementation of this method is difficult because there must be a function which divides and reassembles packets. Furukawa et al. constructs the 
multi-wavelength OPS network with this method using LN switches[8]. They also constructed the implementation of the edge node with the function which converts Ethernet frames into multi-wavelength optical packets. In this paper, we realize multi-wavelength OPS by this method on PLZT switches.

\section{$3 \quad$ Experiment Setup and Detail}

This section describes the setup and its detail of the experiment for confirming the feasibility of the multi-wavelength OPS network using PLZT switches.

The characteristics of PLZT switches are the most suitable for the multiwavelength OPS network. The switching speed of SOA, LN, and PLZT switches is fast enough to perform optical packet switching. However, Optical Signal-Noise Ratio(OSNR) of SOA switches is low due to its high Amplified Spotaneous Emission(ASE) noise. On the other hand, LN switches provides even faster switching speed than one of SOA switches but it has polarization dependency. High drive voltage lowers the dependency but it becomes difficult to drive electric circuits of the switch. In addtion, the LN switches causes DC drift phenomena that its refraction index becomes unstable with direct current. Unlike LN and SOA switches, PLZT switch provides noise tolerance with high OSNR, polarization independence, and operation with low voltage[9].

OPS node mainly consists of four components: 1) the label processing unit which analyzes the destination of packets and determines the output port for the packet, 2) the routing control unit which exchanges routing information with other nodes and calculates the optimized routes, 3) the switching unit which transmits incoming optical packets into the particular port indicated by the label processing unit, and 4) the contention resolution unit which schedules and buffers out-going packets to the same port in order to avoid the packet collisions.

In order to evaluate the feasibility and the performance of the multi-wavelength OPS network, we develop 1) the label processing unit and 3) the switching unit.

\subsection{Detail of Subsystems for Evaluation of The Multi-wavelength Optical Packet Switching Node Performance}

The system for experiments mainly consists of three sub-systems: 1) the payload generation sub-system, 2) the label generation sub-system, and 3) the monitoring sub-system as illustrated in Fig.6.

The payload generation sub-system consists of six main devices generating the signal of the payload and five supplementary devices coordinating the signal. The main devices include the light source (LS), the arrayed waveguide grating (AWG), the $\mathrm{LiNbO}_{3}$ modulator (LNM), the pulse pattern generator (PPG), the AO modulator (AOM), and the Field Programmable Gate Arrays(FPGA). The supplementary devices includes the polarization controller (PC), the polarization maintainer fiber (PMF), the polarization beam splitter (PBS), the EDFA, and the band pass filter(BPF). 


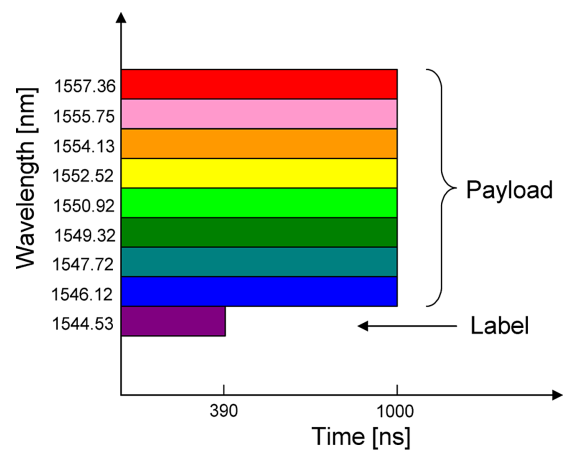

Fig. 5. Packet format: the payload length is $1000 \mathrm{~ns}$

The payload generation sub-system works as follows. First, the LS generates eight continuous waves $(\mathrm{CWs})$, which are independent wavelengths ranging between $1546.12 \mathrm{~nm}$ and $1557.36 \mathrm{~nm}$ at $200 \mathrm{GHz}$ intervals. Then, after the polarization, the signal of each wavelength is coordinated by the PC and the PMF. The AWG multiplexes eight signals into a single optical signal. The polarization of the signal is adjusted to suitable one for the LNM by the PBS. After the PPG and the LNM modulate the signal into the $10 \mathrm{~Gb} / \mathrm{s}$ NRZ signal $\left(2^{7}-1\right.$ PRBS $)$, the power of the modulated signal gains by the EDFA and the noise of the signal is lowered by the BPF. Finally, the AOM cut the signal into impulses of $1000 \mathrm{~ns}$ or 1500 ns. These impulses are treated as the payload.

The label generation sub-system consists of the LS, the AOM, and the FPGA. The LS generates CW of $1544.53 \mathrm{~nm}$ and the AOM controlled by the FPGA modulates the CW into $7.69 \mathrm{Mb} / \mathrm{s} \mathrm{NRZ}$ as a label. Through this experiment, the label is one of two labels: "111" and "100".

The monitoring sub-system consists of the two AWGs and the oscilloscope. The oscilloscope shows the input and output signals for each wavelength.

The arrival interval of the packet is $2500 \mathrm{~ns}$. The packet format with $1000 \mathrm{~ns}$ length is illustrated in Fig.5 and the parameters for the experiment are shown in Table.1.

\subsection{Details of Main Components for Multi-wavelength Optical Packet Switching}

The diagram of this experiment is illustrated in Fig.6.

The label processing sub-system consists of the Photo Detector (PD) and the FPGA. The PD converts an optical signal of the label into an electric signal. The optical signal of the label is received from the label generation sub-system. The FPGA analyzes the electric signal and recognizes the encoded label. To analyze the encoded label we used perfect matching method. Then, FPGA determines the output port for transmission and notifies switching sub-system about the determined output port. 
Table 1. Parameters of multi-wavelength optical packets

\begin{tabular}{|c|c|c|}
\hline & Label & Payload \\
\hline Bit rate & $7.69 \mathrm{Mbps}$ NRZ & 10Gbps NRZ \\
\hline Data length & $390 \mathrm{~ns}$ & $1000 \mathrm{~ns}$ or $1500 \mathrm{~ns}$ \\
\hline Data signal & "111" or "100" & $2^{7}-1$ PRBS \\
\hline Number of wavelength & 1 & 8 \\
\hline Range of wavelength & $1544.53 \mathrm{~nm}$ & $1546.12 \mathrm{~nm} \sim 1557.36 \mathrm{~nm}$ \\
\hline
\end{tabular}

The switching sub-system, which consists of the $1 \times 2$ PLZT switch, transmits the multiplexed payload to the port indicated in the control signal from the label processing sub-system. In this experiment, the switching sub-system transmits the payload with the label " 111 " to the port 1 and one with the label " 100 " to the port 2 .

\section{Experimental Result}

Fig.7 shows the results received with the monitoring sub-system. Fig.7(a), (b), and (c) represent the input signals, the output signals from the port 1, and the output signals from the port 2 , respectively. The signal on the top of the figure represents a signal of the wavelength assigned to the label. The rest of the signals represent the wavelengths of payload fragments. This results show that the packet encoded into multiple wavelengths is correctly switched in accordance with the label information. Therefore, this experiment has confirmed that the multi-wavelength OPS network can be achieved using a PLZT switch.

In addition to the fundamental experiment for the multi-wavelength OPS network, this experiment evaluates the signal degradation coming out of the PLZT switch. The eye patterns of the input and output signals at $1557.36 \mathrm{~nm}$ are shown in Fig.8. The eye patterns of the rest of the wavelengths were similar to patterns presented in Fig.8.

In addition, this experiment measures Bit Error Rate (BER) for all the wavelengths. Fig.9 shows the results of the signal at $1557.36 \mathrm{~nm}$. This results shows that the BER of the output ports was less than $10^{-} 10$ when the input power was $-27 \mathrm{dBm}$. This proposes that this experimental system can perform error-free transmission with high input power.

\section{Conclusions}

In this paper, we have achieved $80 \mathrm{~Gb} / \mathrm{s}$ multi-wavelength OPS using a PLZT switch for evaluation of its feasibility. Moreover, we have confirmed that errorfree transmission is possible in this multi-wavelength OPS node model.

As a future work, we plan to solve the problem caused by group velocity dispersions. Also, we plan to construct $640 \mathrm{~Gb} / \mathrm{s}(40 \mathrm{~Gb} / \mathrm{s} \times 16$ wavelengths $)$ multiwavelength OPS system. 
Acknowledgments. We would like to thank Dr. M. Ohta, Dr. H. Harai, and Dr. N. Wada for their encouragements, discussions, and advices. This reseach is supported by NICT(National Institute of Information and Communicat ions Technology).

\section{References}

1. Mukherjee, B.: WDM Optical Communication Networks: Progress and Challenges. IEEE JOURNAL ON SELECTED AREAS IN COMMUNICATIONS, Vol. 18, No. 10, (2000) 1810-1824 Springer-Verlag, Berlin Heidelberg New York (1997) 415-438

2. Fukuchi, K., Kasamatsu. T., Morie, M., Ohhira, R., Ito, R., Sekiya, K., Ogasahara, D., and Ono, R.: 10.92-Tb/s $(273 \times 40-\mathrm{Gb} / \mathrm{s})$ triple-band/ultra-dense WDM optical-repeatered transmission experiment. Optical Fiber Communication Conference and Exhibit 2001, Vol. 4, PD24, (2001) 1-3

3. Hunter, D.K., Andonovic, I.: Approaches to optical internet packet switching. IEEE Communications Magazine, Vol. 38, No. 2, (2000) 116-122

4. Carena, A., Feo, V.D., Finochietto, J.M., Gaudino, R., Neri, F., Piglione, C., and Poggiolini, P.: RingO: an experimental WDM optical packet network for metro applications. IEEE JOURNAL ON SELECTED AREAS IN COMMUNICATIONS, Vol. 22, No. 8, (2004) 1561-1571

5. Takara, H., Ohara, T. Yamamoto, T. Masuda, H. Abe, M. Takahashi, H. Morioka, T.: Field demonstration of over 1000-channel DWDM transmission with supercontinuum multi-carrier source. Electronics Letters, Vol. 41, (2005) 270-271

6. Onaka, H., Aoki, Y., Sone, K., Nakagawa, G., Kai, Y., Yoshida, S., Takita, Y., Morito, K., Tanaka, S., and Kinoshita, S.: WDM Optical Packet Interconnection using Muti-Gate SOA Switch Architecture for Peta-Flops Ultra-High-Performance Computing Systems. European Conference on Optical Communication 2006, Vol. ${ }^{* *},(2006) * *+* *$

7. Ohta, M..: Components for All Optical Data Path Routers. TECHNICAL REPORT OF IEICE, PN11, (2005)

8. Furukawa, H., Wada, N., Harai, H., Naruse, M., Otsuki, H., Katsumoto, M., Miyazaki, T., Ikezawa, K., Toyama, A., Itou, N., Shimizu, H., Fujinuma, H., Iiduka, H. Cincotti, G., Kitayama, K.: All-Optical Multiple-Label-Processing Based Optical Packet Switch Prototype and Novel 10Gb Ethernet / 80 (8 Lambda x 10) Gbps-Wide Colored Optical Packet Converter with 8-Channel Array Burst-Mode Packet Transceiver. to be presented at Optical Fiber Communication Conference and Exhibit 2007, (2007)

9. Nashimoto, K., Tanaka, N., LaBuda, M., Ritums, D., Dawley, J., Raj, M., Kudzuma, D., and Vo, T.: High-speed PLZT optical switches for burst and packet switching. 2nd International Conference on Broadband Networks, Vol. 2, (2005) $1118-1123$ 


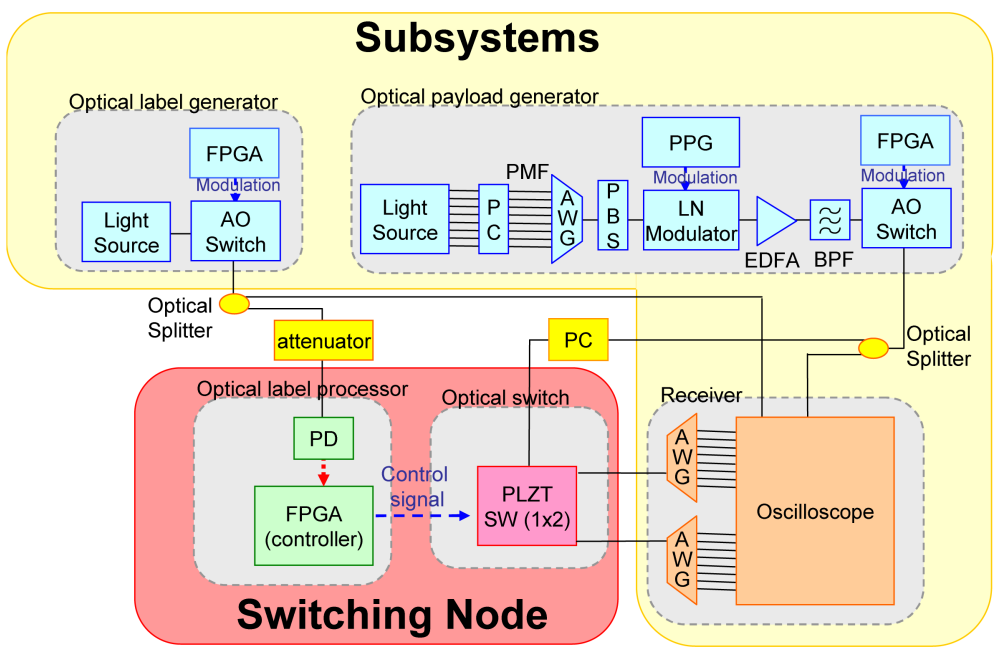

Fig. 6. Experimental Block Diagram

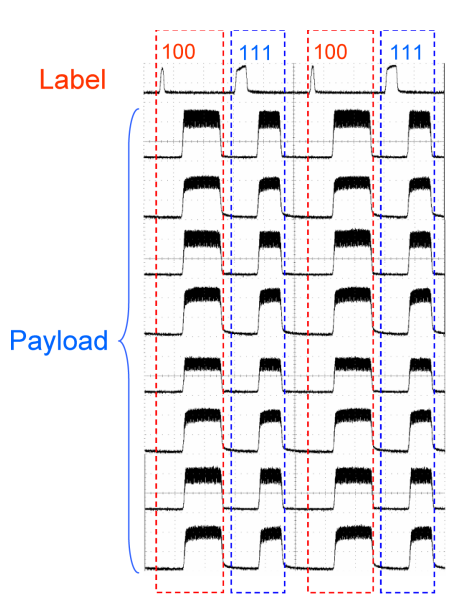

(a) Input

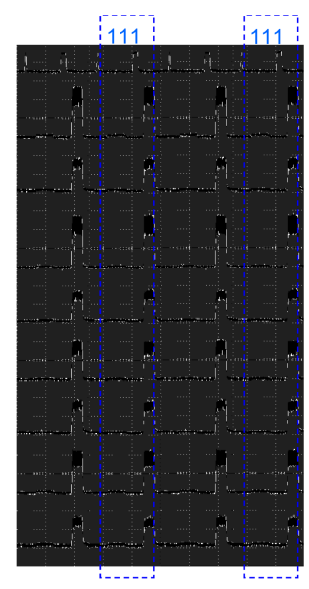

(b) Output-port1

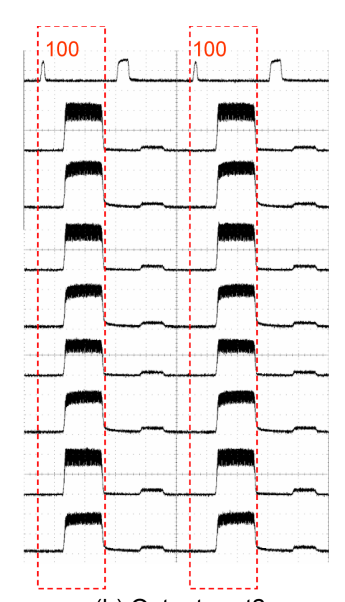

(b) Output-port2

Fig. 7. Input signal \& Output signal

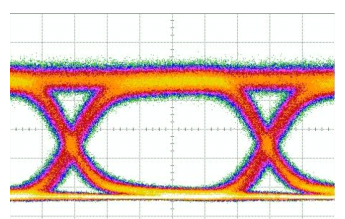

(a) Input

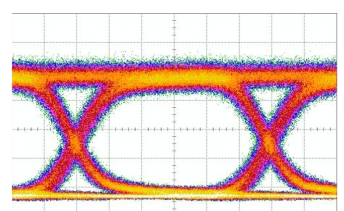

(b) Output-port1

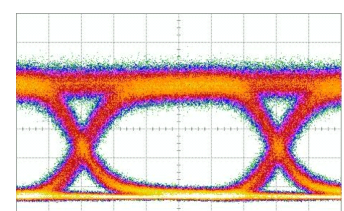

(c) Output-port2

Fig. 8. Eye Pattern diagram @ $1557.36 \mathrm{~nm}$ 
K. Watabe et al.

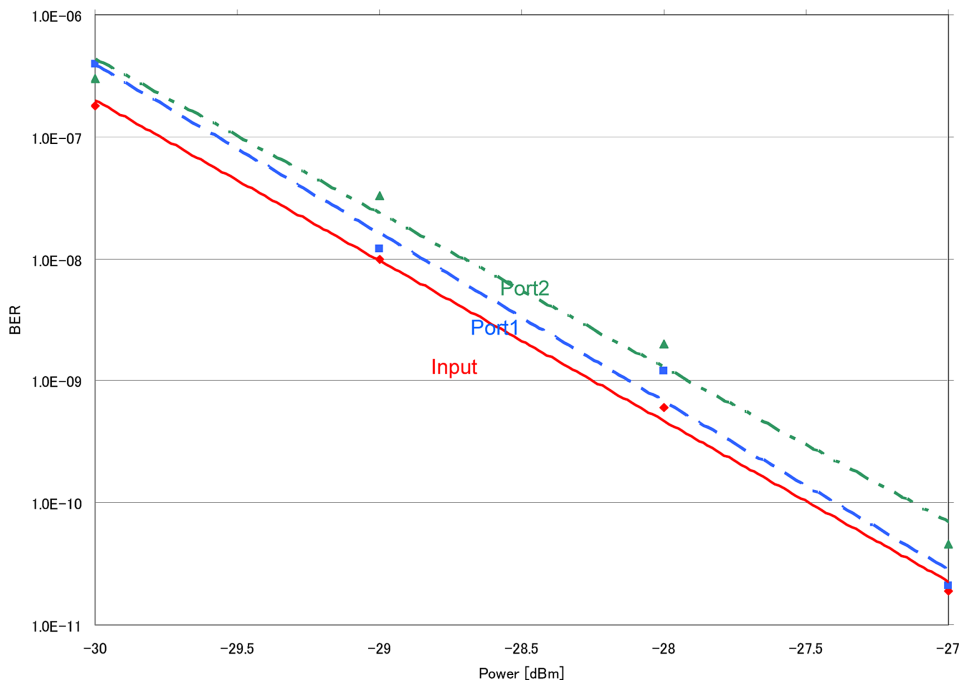

Fig. 9. BER@1557.36nm 